FEATURED CLINICAL CASE

\title{
Platypnoea-orthodeoxia syndrome
}

\author{
P Kubler, H Gibbs, P Garrahy
}

\begin{abstract}
Platypnoea-orthodeoxia is a rare syndrome of postural hypoxaemia accompanied by breathlessness. The predominant symptom, dyspnoea induced by upright posture, can be debilitating and difficult to discern without thorough evaluation of the patient's pattern of dyspnoea. The precise cause of the syndrome is unclear but patients develop right to left intracardiac shunting in the presence of normal right sided cardiac pressures. Initially, patients should have confirmation of orthostatic desaturation by erect and supine pulse oximetry. However, definitive diagnosis of an orthostatic intracardiac shunt is most readily established by echocardiography. The use of echocontrast with postural manoeuvres may facilitate the diagnosis. The treatment of choice is surgical closure of the intracardiac (usually interatrial) communication, which may result in dramatic symptomatic and haemodynamic improvement. Three cases (a 27 year old man and two women aged 63 and 72 years) are described that exemplify the presentation of this syndrome, and reflect the varied management strategies and outcomes of this condition.

(Heart 2000;83:221-223)
\end{abstract}

Keywords: platypnoea-orthodeoxia; echocardiography; intracardiac shunt

Platypnoea-orthodeoxia is an uncommon syndrome of dyspnoea induced by upright posture, with associated arterial hypoxaemia, which is subsequently relieved by recumbency. ${ }^{1}$ The syndrome occurs when there is right to left shunting of blood, usually via an interatrial communication, in the presence of normal pulmonary artery pressure. This is an unusual situation given that most cases of significant right to left intracardiac shunting of blood are associated with increased right sided cardiac pressures. ${ }^{2}$ The three cases described here exemplify the presentation of this syndrome, and reflect the varied management strategies and outcomes of this condition.

\section{Case 1}

A 27 year old man with a known atrial septal defect (ASD) presented with 24 hours of extreme dyspnoea at rest with a history of four months of worsening exertional dyspnoea and lethargy. The ASD had been diagnosed at 9 years of age but the patient had failed to attend follow up care. He had no other significant medical history but was a smoker with a 12 pack-year history of consumption. He had no symptoms suggestive of parenchymal lung disease, pulmonary infections, obstructive sleep disorders or thromboembolic disease. On physical examination his temperature was $37.5^{\circ} \mathrm{C}$, he had a regular pulse at 88 beats $/ \mathrm{min}$, respiration rate $24 / \mathrm{min}$, and blood pressure was $110 / 70 \mathrm{~mm} \mathrm{Hg}$. There was central cyanosis and digital clubbing with two subungual haemorrhages. Lung fields were clear to auscultation, and cardiac examination revealed fixed wide splitting of the second heart sound. There were no regurgitant heart murmurs or palpable precordial impulses. With the patient breathing room air, oxygen saturation was $92 \%$ supine and $84 \%$ in the upright position. Subsequent investigation revealed haemoglobin of $183 \mathrm{~g} / \mathrm{l}$, normal pulmonary ventilation perfusion scan, and normal respiratory function tests. Chest roentgenogram revealed a generalised increase in pulmonary vascular markings without dilatation of the main pulmonary artery or enlargement of the right atrium and ventricle. His ECG showed a right axis deviation with right bundle branch block. Blood cultures remained negative to day 5 .

Echocardiography (transthoracic and subsequently transoesophageal) with bubble contrast study showed a significant right to left shunt at the atrial level through a secundum defect (fig 1). The calculated pulmonary artery pressure was normal (mean $14 \mathrm{~mm} \mathrm{Hg}$ with a peak systolic pulmonary artery pressure of $19-23 \mathrm{~mm} \mathrm{Hg}$ ) with a moderately dilated right atrium and ventricle. Left ventricular size and function were preserved. There was a broad jet of inferior vena caval blood directed towards the ASD. No vegetations were seen.

Subsequent right and left heart catheterisation showed a large ASD (secundum type) with bidirectional shunting of blood. The pulmonary artery pressures were normal $(22 \mathrm{~mm} \mathrm{Hg} /$ $8 \mathrm{~mm} \mathrm{Hg}$, mean $14 \mathrm{~mm} \mathrm{Hg}$ ) as was the coronary artery anatomy. The patient underwent open surgical closure of the ASD with a patch applied to the $3 \mathrm{~cm}$ diameter defect. He had an uneventful recovery and remained asymptomatic at four years' follow up. Furthermore, the clubbing resolved as did the hypoxaemia.

\section{Case 2}

A 63 year old woman presented with a six week history of worsening exertional dyspnoea with 

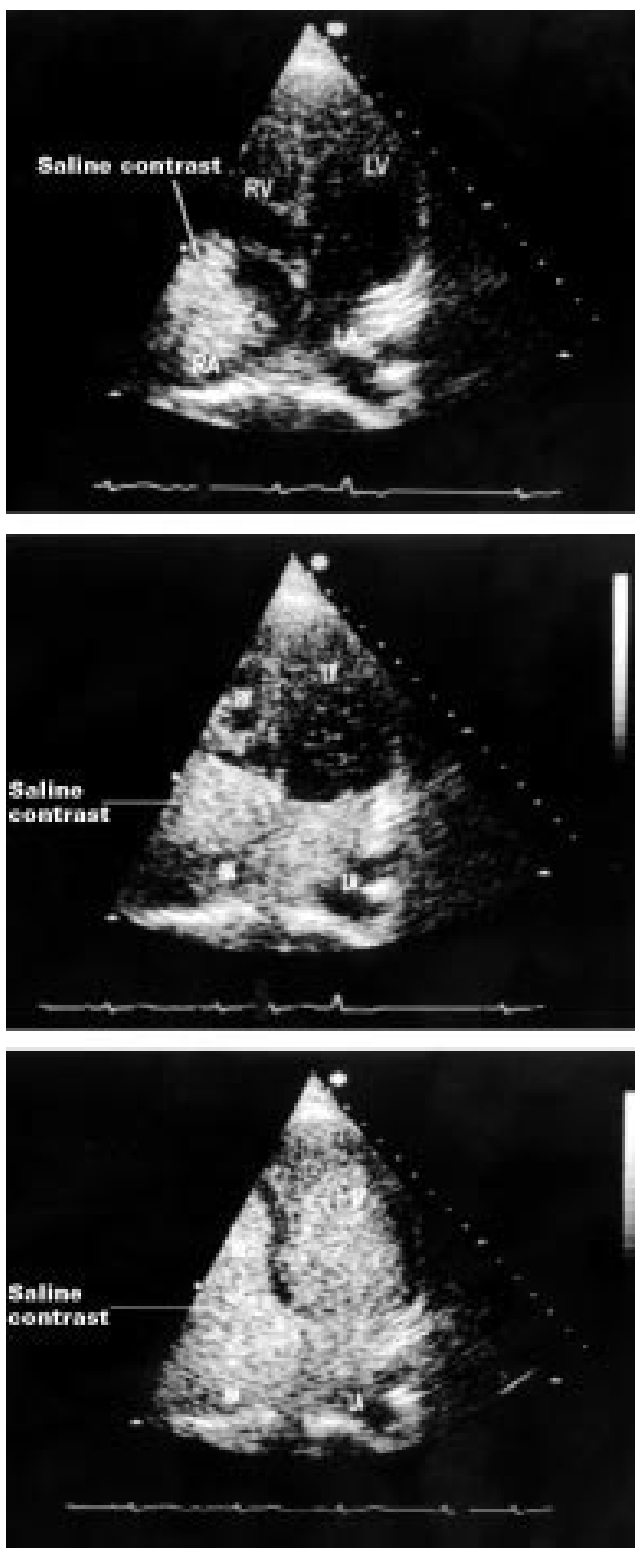

Figure 1 Sequential apical four chamber echocardiographic frames of case 1 showing transit of saline contrast soon after injection into left side of heart via an interatrial defect. $L A$, left atrium; $R A$, right atrium; $L V$, left ventricle; $R V$, right ventricle.

reduction in exercise tolerance to less than 50 metres. She denied any associated anginal symptoms and remarked how the breathlessness abated quickly with recumbency. Six months previously, she had undergone coronary artery bypass grafting for anginal symptoms refractory to medical treatment. Her postoperative course had been complicated by a right occipital and thalamic infarct (confirmed by magnetic resonance imaging) with a residual left homonymous hemianopia, left hemisensory disturbance, and significant tremor with gait abnormality. On physical examination, values for blood pressure, pulse, and respiratory rate were within normal limits. There was no cyanosis or digital clubbing and heart sounds were of normal intensity with physiological splitting and without audible murmur. The lung fields were clear to auscultation and the remainder of the examination was normal apart from the neurological abnormalities. Oxygen saturation was $98 \%$ with the patient supine and $74 \%$ while standing. Routine haematology and biochemical blood analysis were normal, as was the resting ECG and chest roentgenogram. Arterial blood gases performed on room air showed a significant hypoxaemia $\left(\mathrm{PO}_{2} 68 \mathrm{~mm} \mathrm{Hg}\right)$ with an alveolar to arterial gradient of greater than $40 \mathrm{~mm} \mathrm{Hg}$. Pulmonary ventilation perfusion scan, noninvasive peripheral vascular studies of the lower limb venous system, and pulmonary function tests were normal.

Transoesophageal echocardiography showed a mobile interatrial septum with no definitive defect. Echocontrast confirmed the presence of right to left shunting of blood with the appearance of left sided bubbles soon after injection. The calculated pulmonary artery pressure was normal (mean $12 \mathrm{~mm} \mathrm{Hg}$, peak systolic pulmonary artery pressure $19-23 \mathrm{~mm} \mathrm{Hg}$ ) and left ventricular systolic function was preserved. There was moderate tricuspid regurgitation with a broad jet of central regurgitant flow directed at the interatrial septum. Subsequent right atriogram confirmed pulsatile shunting from the right atrium to the left atrium across the superior limb of the foramen ovale membrane. Coronary angiography revealed no stenosis in the grafted vessels.

Following consultation with a cardiac surgeon, the patient declined any surgical intervention given her previous complicated cardiac surgery. Her symptoms have not progressed in two years of follow up, however the ability to perform many activities of daily living have been significantly curtailed by exertional and postural dyspnoea.

\section{Case 3}

A 72 year old woman with a 10 year history of schizophrenia, for which she had been an inpatient for one month with acute psychosis, was noted by a duty nurse to appear breathless and cyanosed on walking to the shower. Her oxygen saturation was $76 \%$ on room air at the time of this observation and she was returned to bed. Review by the duty medical officer showed values for blood pressure, pulse, and respiratory rate were within normal limits. Oxygen saturation in the supine position was $98 \%$. Cardiorespiratory examinations were unremarkable apart from a soft $2 / 6$ ejection systolic murmur along the left sternal border, which radiated into the carotids. The patient was non-communicative owing to her psychosis and as such no symptoms were obtainable. Routine haematological and biochemical blood analysis were normal as was her resting ECG. Chest roentgenogram revealed a tortuous proximal aorta with clear lung fields. Computed tomography pulmonary angiography was normal apart from a $5 \mathrm{~cm}$ diameter aneurysm of the ascending aorta. Syphilis serology was negative. Non-invasive peripheral venous studies of the lower limbs were also negative.

The observed breathlessness and cyanosis were reproducible on subsequent mobilisation and consequently the patient underwent transthoracic echocardiography with bubble contrast 
study. This revealed mild aortic stenosis and significant right to left shunting of blood through a patent foramen ovale in the presence of normal pulmonary artery pressure (calculated mean $12 \mathrm{~mm} \mathrm{Hg}$, peak systolic pulmonary artery pressure 19-23 mm Hg). The patient died suddenly four hours after echocardiography. Necropsy revealed the cause of death to be rupture of a thoracic atherosclerotic aortic aneurysm; a large probe patent foramen ovale $2 \mathrm{~cm}$ in diameter was also evident.

\section{Discussion}

Platypnoea-orthodeoxia is an uncommon syndrome of dyspnoea resulting from arterial hypoxaemia, which is accentuated by the upright position and relieved by recumbency. This syndrome may occur in patients with an intracardiac shunt, usually at the atrial level, or with a history of lung disease such as severe obstructive lung disease, post-pneumonectomy and pulmonary vascular shunting. ${ }^{1}$ The right to left shunting of blood through an interatrial defect occurs in the setting of normal pulmonary artery pressures, and this is unusual given that most cases of right to left intracardiac shunting have associated increased right sided intracardiac pressure. ${ }^{2}$

We present three cases, each with an interatrial defect who presented with platypnoea and orthodeoxia. By December 1998, this syndrome had only been reported in the medical literature in 20 cases. $^{34}$ The predominant symptom of the syndrome, postural dyspnoea, can be debilitating as occurred in the three cases we report. The precise mechanisms for both platypnoea and orthodeoxia are unknown, as is the apparent rarity of the syndrome, given that the most common aetiological association, an interatrial communication, is common; an estimated $25 \%$ of the general population having a patent foramen ovale. ${ }^{5}$

Various theories have been advanced to explain why patients with a patent foramen ovale may develop this syndrome. Explanations include preferential blood flow directed towards the atrial septum, which can be accentuated by altered intracardiac anatomical relations, unequal diastolic compliance between the right and left sides of the heart, and transient right to left pressure gradients associated with respiratory manoeuvres, all of which can result in significant right to left shunting in the upright position. The patients described in cases 1 and 2 both had echocardiographic evidence of preferential bloodflow directed towards the interatrial septum, which may have been a significant factor in the production of right to left shunting in these cases. In case 1, inferior vena caval inflow was directed towards an ASD. While inferior vena caval blood is often directed towards the interatrial septum, accentuation of this may result from either a persistent, abnormally large Eustachian valve at the junction of the inferior vena cava and right atrium, or from anatomical distortion of the heart with subsequent displacement of the right atrium and interatrial septum. ${ }^{6}$ In case 2 , a broad jet of tricuspid regurgitant flow was present directed towards a patent foramen ovale. Preferential flow of blood toward the interatrial septum owing to tricuspid regurgitation has not previously been described in association with platypnoea-orthodeoxia. Case 3 developed the platypnoea-orthodeoxia syndrome in association with a proximal aortic aneurysm.

Three similar cases have been described in the literature. ${ }^{67}$ It has been suggested that enlargement of the ascending aorta may rotate the heart counterclockwise and distort the position of the atrial septum causing a patent foramen ovale to receive preferential venous inflow in the erect position.

Platypnoea-orthodeoxia syndrome should be considered in the differential diagnosis of positional dyspnoea and hypoxaemia out of proportion to any underlying cardiopulmonary disease, and may be a difficult diagnosis to make unless there is a high index of suspicion. Among dyspnoeic patients, discernment of a pattern of platypnoea and orthodeoxia is the key to effective evaluation. Initially orthostatic desaturation should be confirmed by erect and supine pulse oximetry, but more definitive diagnosis of a posturally related right to left shunt can be established by contrast echocardiography with postural manoeuvres. ${ }^{8}$ However, in some patients - and before surgical correction-right and left cardiac catheterisation will be required.

The treatment of choice is surgical closure of the intracardiac communication. As demonstrated in case 1 , this can result in a dramatic symptomatic improvement, as well as eliminating the site of a possible paradoxical embolus. ${ }^{6}$ However, in some patients, such as case 2 , surgery carries a significant risk.

Platypnoea-orthodeoxia is a rare condition with the potential for surgical cure. Noninvasive investigations can readily establish the diagnosis and should be performed when this condition is suspected.

1 Seward JB, Hayes DL, Smith HC, et al. Platypneaorthodeoxia: clinical profile, diagnostic workup, management, and report of 7 cases. Mayo Clin Proc 1984;59:22131

2 Nootens MT, Beraarducci LA, Kaufmann E, et al. The prevalence and significance of a patent foramen ovale in pulmonary hypertension. Chest 1993;104:1673-75.

3 Robin E, McCauley R. An analysis of platypneaorthodeoxia syndrome including a "new" therapeutic approach. Chest 1997;112:1449-51.

4 Danner I. A rare dyspnea in internal medicine: platypneaorthodeoxia after pneumonectomy. Rev Med Intern 1998; 29:76-9.

5 Hagen PT, Scholz DG, Edwards WD. Incidence and size of patent foramen ovale during the first 10 decades of life: an autopsy of 965 normal hearts. Mayo Clin Proc 1984;59:1720

6 Popp G, Melek H, Garnett AR. Platypnea-orthodeoxia related to aortic elongation. Chest 1997;112:1682-4.

7 Laybourn KA, Martin ET, Cooper RA, et al. Platypnea and orthodeoxia: shunting associated with an aortic aneurysm. 7 Thorac Cardiovasc Surg 1997;113:955-6.

8 Khouzaie TA, Busser JR. A rare cause of dyspnea and arterial hypoxemia. Chest 1997;112:1681-2. 